\title{
Listeria monocytogenes endophthalmitis following keratoconjunctivitis
}

This article was published in the following Dove Press journal:

Clinical Ophthalmology

24 January 2014

Number of times this article has been viewed

\author{
Samir S Shoughy' \\ Khalid F Tabbara ${ }^{1-3}$ \\ IThe Eye Center and The Eye \\ Foundation for Research in \\ Ophthalmology, Riyadh, Saudi Arabia; \\ ${ }^{2}$ Department of Ophthalmology, \\ College of Medicine, King Saud \\ University, Riyadh, Saudi Arabia; ${ }^{3}$ The \\ Wilmer Ophthalmological Institute of \\ The Johns Hopkins University School \\ of Medicine, Baltimore, MD, USA
}

\begin{abstract}
Endophthalmitis due to endogenous or exogenous bacteria is a rare infection of the eye. We report a case of endophthalmitis following Listeria monocytogenes keratoconjunctivitis in a 27 -year-old healthy white male presenting with hand motion visual acuity, right eye mucopurulent conjunctivitis, elevated intraocular pressure, and pigmented hypopyon 6 months post-keratectomy. The conjunctivitis was unresponsive to a 5-day course of topical tobramycin eye drops, and the patient developed keratitis with pain that progressed to endophthalmitis after 21 days. Diagnostic B-scan revealed vitreous exudates. Intraocular fluid specimen showed Gram-positive organisms and the aqueous culture grew penicillin-/aminoglycoside-sensitive L. monocytogenes. The patient was given intravitreal and systemic vancomycin and ceftazidime. The eye was unresponsive to intravenous penicillin and gentamicin; the anterior chamber progressively flattened and developed phthisis bulbi. L. monocytogenes keratoconjunctivitis may lead to bacterial endophthalmitis. Prompt culture and early antibiotic therapy are recommended.
\end{abstract}

Keywords: conjunctivitis, L. monocytogenes, endophthalmitis

\section{Introduction}

Listeria monocytogenes is a pathogenic Gram-positive bacterium. It is frequently overlooked as a cause of illness due to its unique growth capabilities. The ability of Listeria to grow in diverse environments is one of the challenges presented by this bacterium. Most bacteria grow poorly when the temperature falls below $4^{\circ} \mathrm{C}$, while Listeria survives in temperatures below freezing and grows best at $-18^{\circ} \mathrm{C}$ to $10^{\circ} \mathrm{C}$, a temperature range that is used for refrigeration. As a result, Listeria may be transmitted in ready-to-eat foods that have been kept properly refrigerated. ${ }^{1}$ It can cause a rare but serious disease called listeriosis, especially among pregnant women, the elderly, or individuals with compromised immune systems. In serious cases, it can lead to brain infection and death. ${ }^{1}$

Ocular listeriosis is rare, with conjunctivitis being the most frequent manifestation. Keratitis, sclerokeratitis, endophthalmitis, and acute chorioretinitis have also been reported..$^{2-5}$

Endogenous endophthalmitis results from the hematogenous spread of bacterial infection to the eye. Endophthalmitis caused by L. monocytogenes is a potentially devastating disease that may lead to loss of vision. ${ }^{6}$

Cases of endophthalmitis caused by L. monocytogenes demonstrate clinical features similar to each other, including decreased vision, elevated intraocular pressure, and pigment dispersion with dark hypopyon. ${ }^{7}$ The presence of a dark
Eye Center, 24l Makkah Road, The Eye Center, 24I Makkah Road, PO Box 55307, Riyadh II534, Saudi Arabia

Email k.tabbara@nesma.net.sa 
hypopyon ascribed to pigment dispersion may indicate intraocular infection with L. monocytogenes, even in an apparently healthy host. ${ }^{7,8}$

Ultrasound biomicroscopy examination of a case of Listeria endophthalmitis showed increased iris thickness with rarefaction of tissue with irregular echogenicity due to both increased thickness and rarefaction of tissue, as well as iris pigment epithelial detachment. The detection of both pigment epithelial detachment and changes in the iris tissue could explain the pigmented hypopyon that frequently develops in Listeria endophthalmitis with significant pigment dispersion. ${ }^{9}$ Pigmented hypopyon has also been reported in the setting of necrotic intraocular melanoma as well as Serratia marcescens endogenous endophthalmitis and Streptococcus bovis endogenous endophthalmitis. ${ }^{10,11}$

L. monocytogenes has also been found to cause fulminant anterior uveitis, confirmed by culture and polymerase chain reaction. $^{12}$

Since the first case of endophthalmitis due to L. monocytogenes, reported by Goodner and Okumoto in $1967,{ }^{13}$ several cases of L. monocytogenes-induced endophthalmitis have been reported. ${ }^{6,12,14}$

Listeria is a fastidious organism that is difficult to grow. Listeria may be confused with common harmless organisms and can be disregarded as a cause of the disease.

Institutional review board approval was obtained and recorded.

\section{Case report}

A 27-year-old healthy white male presented with history of mucopurulent conjunctivitis that was treated with topical tobramycin eye drops for 5 days prior to presentation. After 21 days, he complained of redness and photophobia in the right eye followed by decrease in vision in the same eye. The patient reported a history of photorefractive keratectomy in both eyes 6 months prior to presentation. There was no history of trauma or contact lens wear.

On eye examination, the best corrected visual acuity in the right eye was hand motion, and in the left eye was 20/20. Intraocular pressure was $18 \mathrm{mmHg}$ in the right eye and $13 \mathrm{mmHg}$ in the left eye. Slit-lamp biomicroscopy of the right eye revealed keratitis with severe corneal edema. The anterior chamber was deep and showed brown pigmented hypopyon. The left eye was normal. Diagnostic B-scan ultrasonography of the right eye was performed and showed exudates and opacification of the vitreous. After 2 days, a vitreous biopsy was done and intravitreal injection of vancomycin $1 \mathrm{mg} / 0.1 \mathrm{~mL}$ and ceftazidime $2.25 \mathrm{mg} / 0.1 \mathrm{~mL}$ were given.
Gram stain showed multiple Gram-positive bacilli. The culture revealed growth that was labeled as contamination.

The patient was kept on topical tobramycin and systemic amoxicillin and clavulanate antibiotics, but there was no improvement. The clinical picture of endophthalmitis started to be established with profound loss of vision, hypopyon, and massive vitritis. Four days following the initial tap, the patient was taken to the operating room again and an anterior chamber paracentesis was done and aqueous specimens obtained. Anterior chamber washout and vitreous biopsy were performed. The patient received intracameral antibiotics comprising vancomycin $1 \mathrm{mg} / 0.1 \mathrm{~mL}$, ceftazidime $2.25 \mathrm{mg} / 0.1 \mathrm{~mL}$, and cefuroxime $1 \mathrm{mg} / 0.1 \mathrm{~mL}$ at that time. Vitreous tap was taken and intravitreal antibiotics comprising vancomycin $1 \mathrm{mg} / 0.1 \mathrm{~mL}$ and ceftazidime $2.25 \mathrm{mg} / 0.1 \mathrm{~mL}$ were given again. The samples were sent for microbiology and polymerase chain reaction. The result from the aqueous tap was positive for L. monocytogenes that was resistant to most drugs except for penicillin and aminoglycosides. The polymerase chain reaction for aqueous and vitreous tap was negative. A diagnosis of L. monocytogenes-induced endophthalmitis was made. The patient was hospitalized and received intravenous penicillin 0.5 million units every 6 hours, and fortified gentamicin drops were applied hourly to the right eye for 7 days. The ocular inflammations subsided. However, eye examinations revealed that the shallow anterior chamber progressively became flat; the cornea remained edematous; the pupil showed $360^{\circ}$ posterior synechiae; the lens was cataractous; and the posterior pole was obscured over the course of 2 weeks. A diagnostic B-scan was performed and showed diffuse thickening of the retinochoroidal layer, but no choroidal detachment (Figure 1). Ultrasound biomicroscopy revealed a thick cornea; lens displacement anteriorly, almost touching the cornea; and a $360^{\circ}$ shallow

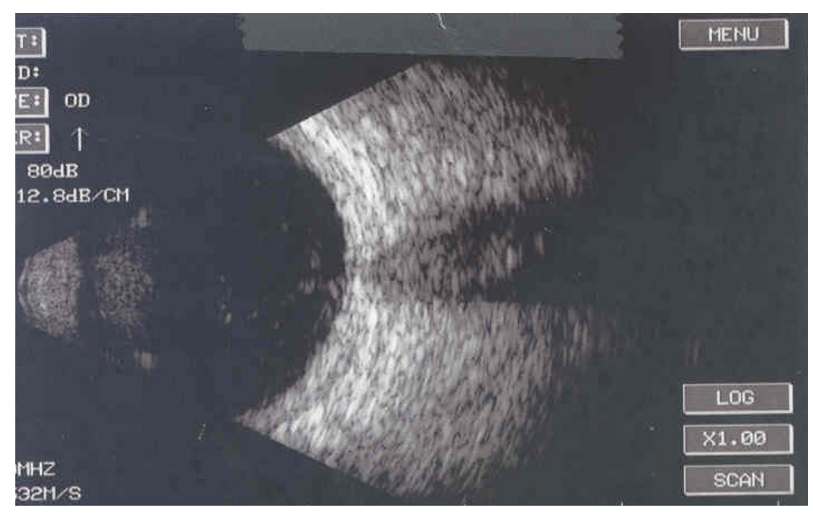

Figure I B-scan ultrasonography of the right eye showing diffuse thickening of the retinochoroidal layer without choroidal detachment. 
ciliary body detachment. The condition progressed and the eye developed phthisis bulbi 4 months after onset.

\section{Discussion}

Most cases of endophthalmitis induced by L. monocytogenes described in the literature ${ }^{17}$ have shown similar clinical features, including decreased vision, elevated intraocular pressure, and fibrinous anterior chamber reaction. In our patient, the intraocular pressure was higher than in the affected eye. Several patients have been reported to have developed a dark hypopyon similar to our patient. ${ }^{7,8}$ In all reported cases, the cause of bacterial endophthalmitis was determined by recovering the organism from cultures of intraocular fluids. ${ }^{2}$

In our case, the initial culture showed multiple Grampositive bacilli, and the growth of the Gram-positive bacilli was labeled as contamination. Listeria can be confused and mistakenly disregarded. ${ }^{1}$ The result from the repeated aqueous tap was positive for L. monocytogenes that was resistant to most drugs except for penicillin and aminoglycosides. Microbiological studies should be interpreted carefully and repeated if necessary.

To date, all cases of $L$. monocytogenes endophthalmitis have been presumed to be endogenous, ${ }^{2,6,7,9,12,14}$ although L. monocytogenes has the ability to penetrate intact conjunctival and corneal epithelium. The pathogenesis of bacterial infection often involves a compromised epithelial surface, because an intact epithelium is an effective barrier to most organisms. However, Neisseria gonorrhoeae, Corynebacterium diphtheriae, Haemophilus aegyptius, and L. monocytogenes can penetrate intact healthy epithelium through specialized attachments, adhesion molecules, pili, or toxins. These attachments allow adhesions that may lead to bacterial tissue invasion. ${ }^{15}$ The ability of $L$. monocytogenes to cause conjunctivitis and keratitis has been well established in animals. ${ }^{16} \mathrm{~L}$. monocytogenes may cause corneal ulcer in patients with diabetes mellitus. ${ }^{17}$ The prognosis of antibiotic therapy is usually poor. ${ }^{17}$

The clinical course of our case started with conjunctivitis secondary to L. monocytogenes. The patient was treated with topical antibiotics. Despite antimicrobial therapy, the patient developed peripheral keratitis and diffuse corneal edema with pain. At this stage of the disease, there was no evidence of endophthalmitis and the fundus view was not obscured. Subsequently, the patient developed hypopyon. He started to have severe pain and marked deterioration of vision. Severe anterior uveitis was noted and progressed into endophthalmitis. The ability of $L$. monocytogenes to penetrate the intact cornea, causing keratitis without ulceration or perforation, may explain the development of anterior uveitis with hypopyon and later endophthalmitis.

Although acute bacterial conjunctivitis is frequently considered to be self-limiting, there are concerns about whether antibiotic therapy confers significant benefit or not. In a recent review of eleven randomized clinical trials with a total of 3,673 participants, it was found that the use of antibiotic eye drops is associated with modestly improved rates of clinical and microbiological remission in comparison to the use of placebo. ${ }^{18}$ The use of antibiotic eye drops should, therefore, be considered in order to induce resolution of symptoms and infection.

\section{Conclusion}

We have reported herein a case of Listeria endophthalmitis that followed conjunctivitis. Prompt diagnosis and early treatment are mandatory to prevent endophthalmitis. To the best of our knowledge, this is the first report of a case of L. monocytogenes-induced endophthalmitis following keratoconjunctivitis. This report emphasizes the importance of appropriate and precise identification of the causative organism in order to manage cases of endophthalmitis effectively. Although endophthalmitis due to L. monocytogenes is uncommon, the treating physician should keep in mind the possibility of L. monocytogenes endophthalmitis regardless of the age or immunological status of the patient. Microbiological studies should be interpreted carefully and repeated if there is a high degree of clinical suspicion of L. monocytogenes-induced endophthalmitis. The ability of Listeria to penetrate intact epithelium may lead to endophthalmitis following keratoconjunctivitis.

\section{Acknowledgment}

This study was supported in part by a Special Fund from The Eye Center and The Eye Foundation for Research in Ophthalmology, Riyadh, Saudi Arabia.

\section{Disclosure}

The authors report no conflicts of interest in this work. The authors alone were responsible for the content and writing of this paper.

\section{References}

1. Ramaswamy V, Cresence VM, Rejitha JS, et al. Listeria - review of epidemiology and pathogenesis. J Microbiol Immunol Infect. 2007;40(1): 4-13

2. Betriu C, Fuentemilla S, Méndez R, Picazo JJ, García-Sánchez J. Endophthalmitis caused by Listeria monocytogenes. J Clin Microbiol. 2001;39(7):2742-2744. 
3. Schuchat A, Swaminathan B, Broome CV. Epidemiology of human listeriosis. Clin Microbiol Rev. 1991;4(2):169-183.

4. Altaie R, Fahy GT, Cormican M. Failure of Listeria monocytogenes keratitis to respond to topical ofloxacin. Cornea. 2006;25(7):849-850.

5. Tay E, Rajan M, Tuft S. Listeria monocytogenes sclerokeratitis: a case report and literature review. Cornea. 2008;27(8):947-949.

6. Otomo K, Kaburaki T, Shigeeda T, Takamoto M, Kawashima H, Araie M. Bilateral anterior uveitis in a patient with bacterial meningitis. Int Ophthalmol. 2012;32(4):401-403.

7. Eliott D, O'Brien TP, Green WR, Jampel HD, Goldberg MF. Elevated intraocular pressure, pigment dispersion and dark hypopyon in endogenous endophthalmitis from Listeria monocytogenes. Surv Ophthalmol. 1992;37(2):117-124.

8. Abbott RL, Forster RK, Rebell G. Listeria monocytogenes endophthalmitis with a black hypopyon. Am J Ophthalmol. 1978;86(5): $715-719$.

9. Mendez-Hernandez C, Garcia-Feijoo J, Garcia-Sanchez J. Listeria monocytogenes-induced endogenous endophthalmitis: bioultrasonic findings. Am J Ophthalmol. 2004;137(3):579-581.

10. Equi RA, Green WR. Endogenous Serratia marcescens endophthalmitis with dark hypopyon: case report and review. Surv Ophthalmol. 2001;46(3):259-268.
11. Hauch A, Eliott D, Rao NA, Vasconcelos-Santos DV, O'Hearn T, Fawzi AA. Dark hypopyon in Streptococcus bovis endogenous endophthalmitis: clinicopathologic correlations. J Ophthalmic Inflamm Infect. 2010;1(1):39-41

12. Hueber A, Welsandt G, Grajewski RS, Roters S. Fulminant endogenous anterior uveitis due to Listeria monocytogenes. Case Rep Ophthalmol. 2010;1(2):63-65.

13. Goodner EK, Okumoto M. Intraocular listeriosis. Am J Ophthalmol. 1967;64(4):682-686.

14. Berger E, Donat M, Guthoff RF, Podbielski A. [Listeria endophthalmitis]. Ophthalmologe. 2005;102(9):888-890. German.

15. Buchanan TM. Surface antigens pili. In: Roberts RB, editor. The Gonococcus. New York: John Wiley and Sons Ltd; 1977:225-272.

16. Evans K, Smith M, McDonough P, Wiedmann M. Eye infections due to Listeria monocytogenes in three cows and one horse. $J$ Vet Diagn Invest. 2004;16(5):464-469.

17. Bialasiewicz AA, Holbach L, Boltze HJ. [Listeria monocytogenes kerato-uveitis]. Klin Monbl Augenheilkd. 1988;193(1):62-65. German.

18. Sheikh A, Hurwitz B, van Schayck CP, McLean S, Nurmatov U. Antibiotics versus placebo for acute bacterial conjunctivitis. Cochrane Database Syst Rev. 2012;9:CD001211.
Clinical Ophthalmology

\section{Publish your work in this journal}

Clinical Ophthalmology is an international, peer-reviewed journal covering all subspecialties within ophthalmology. Key topics include: Optometry; Visual science; Pharmacology and drug therapy in eye diseases; Basic Sciences; Primary and Secondary eye care; Patient Safety and Quality of Care Improvements. This journal is indexed on Submit your manuscript here: http://www.dovepress.com/clinical-ophthalmology-journal

\section{Dovepress}

PubMed Central and CAS, and is the official journal of The Society of Clinical Ophthalmology (SCO). The manuscript management system is completely online and includes a very quick and fair peer-review system, which is all easy to use. Visit http://www.dovepress.com/ testimonials.php to read real quotes from published authors. 